\begin{tabular}{c} 
International Journal of Engineering \& Technology, 7 (4.36) (2018) 813-816 \\
International Journal of Engineering \& Technology \\
SPC \\
Website: www.sciencepubco.com/index.php/IJET \\
Research paper \\
\hline
\end{tabular}

\title{
Einstein Operations of Fuzzy Matrices
}

\author{
T.M. Selvarajan ${ }^{1 *}$, S. Sriram ${ }^{2}$, R.S. Ramya ${ }^{3}$ \\ ${ }^{1}$ Department of Mathematics, Noorul Islam University, Kanyakumari, India. \\ ${ }^{2}$ Department of Mathematics, Annamalai University, Chidambaram, India. \\ ${ }^{3}$ Department of Mathematics, Noorul Islam University, Kanyakumari, India.
}

\begin{abstract}
In this paper, the authors defined the Einstein operations of fuzzy matrices, intuitionistic fuzzy matrices and proved several properties of them.
\end{abstract}

Keywords: Fuzzy matrix, intuitionistic fuzzy matrix einstein sum and einstein product.

\section{Introduction}

It is outstanding that lattices assume significant job in different zones, for example, Arithmetic, Material science, Measurements, Designing, Sociologies and numerous others. Be that as it may, we can't effectively utilize established lattices in view of different kinds of vulnerabilities present in certifiable circumstances. Presently a days likelihood, Fluffy sets, Intuitionistic fluffy sets, Unclear sets are utilized as Numerical instruments for managing vulnerabilities. Fluffy lattices emerge in numerous applications, one of which is as nearness frameworks of fluffy relations. A fluffy network is a framework over the fluffy variable based math $\mathfrak{I}=[0,1]$ under the fluffy activities defined by Zadeh in 1965 [8]. A few creators displayed various outcomes on fluffy grids. In 1977, Thomoson [6] examined the conduct of forces of fluffy networks utilizing max-min sythesis. The hypothesis of fluffy lattices was efficiently created by Kim and Roush in [2]. Ragab and Emam [4] contemplated a few properties of the min-max sytheses of fluffy networks; it tends to be viewed as the double of max-min piece of fluffy lattices .Among the outstanding activities which can be performed on fluffy frameworks are the tasks $\vee, \wedge$ and complementation. Notwithstanding these tasks, the activities $\bigoplus$ and $\odot$ are presented by Shyamal and Buddy [5]. Additionally a few properties on $\oplus$ and $\odot$, a few outcomes on existing administrators alongside these activities are examined. Wang and Liu[7] presented some Einstein activities of intuitionistic fluffy sets and break down some alluring properties of the proposed tasks. In this paper, we stretch out the Einstein activities to fluffy lattices and demonstrated a few properties of them.

\subsection{Fuzzy matrices}

Definition: If $A$ and $B$ are two fuzzy matrices of same size, where $A=\left[a_{i j}\right]$ and $B=\left[b_{i j}\right]$ then

(i) The Einstein sum of $A$ and $B$ is defined by $A \oplus_{\varepsilon} B=$ $\left[\frac{a_{i j}+b_{i j}}{1+a_{i j} b_{i j}}\right]$

(ii) The Einstein product of $A$ and $B$ is defined by

$$
A \odot_{\varepsilon} B=\left[\frac{a_{i j} b_{i j}}{1+\left(1-a_{i j}\right)\left(1-b_{i j}\right)}\right] .
$$

Property 1.1: If $A$ and $B$ are two fuzzy matrices of same size, then $A \oplus_{\varepsilon} B \geq A \bigodot_{\varepsilon} B$.

Proof: The $i j^{\text {th }}$ element of $A \bigoplus_{\varepsilon} B$ is $\frac{a_{i j}+b_{i j}}{1+a_{i j} b_{i j}}$ and that

$$
\begin{aligned}
& \text { of } A \bigodot_{\varepsilon} B \text { is } \frac{a_{i j} b_{i j}}{1+\left(1-a_{i j}\right)\left(1-b_{i j}\right)} . \\
& \text { Since } a_{i j}+b_{i j} \geq \\
& \quad 2 a_{i j} b_{i j} \\
& =a_{i j} b_{i j}+a_{i j} b_{i j} \\
& \geq a_{i j} b_{i j}+\left(a_{i j} b_{i j}\right)^{2} \\
& =a_{i j} b_{i j}\left(1+a_{i j} b_{i j}\right) \\
& \frac{a_{i j}+b_{i j}}{\left(1+a_{i j} b_{i j}\right)} \\
& \geq a_{i j} b_{i j}---(1.1)
\end{aligned}
$$

Also $a_{i j} b_{i j} \geq \frac{a_{i j} b_{i j}}{1+\left(1-a_{i j}\right)\left(1-b_{i j}\right)}$, as $1+\left(1-a_{i j}\right)(1-b) \geq 1-$ $---(1.2)$

From (1.1) and (1.2) $\quad \frac{a_{i j}+b_{i j}}{\left(1+a_{i j} b_{i j}\right)} \geq \frac{a_{i j} b_{i j}}{1+\left(1-a_{i j}\right)\left(1-b_{i j}\right)}$ for all $i$ and $j$.

Hence, $A \oplus_{\varepsilon} B \geq A \odot_{\varepsilon} B$.

Property1. 2: For any fuzzy matrix $A$,

(i) $A \oplus_{\varepsilon} A \geq A$

(ii) $A \bigodot_{\varepsilon} A \leq A$.

Proof: $(i)$ The $i j^{\text {th }}$ element of $A \bigoplus_{\varepsilon} A$ is $\frac{2 a_{i j}}{1+a_{i j}^{2}}$

Since $2 \geq\left(1+a_{i j}^{2}\right)$

$$
\begin{gathered}
2 a_{i j} \geq a_{i j}\left(1+a_{i j}^{2}\right) \\
\frac{2 \mathrm{a}_{\mathrm{ij}}}{1+\mathrm{a}_{\mathrm{ij}}^{2}} \geq a_{i j}, \text { for all } i \text { and } j .
\end{gathered}
$$

Hence, $A \oplus_{\varepsilon} A \geq A$.

(ii) The $i j^{\text {th }}$ element of $A \bigodot_{\varepsilon} A$ is $\frac{\boldsymbol{a}_{i j}^{2}}{\mathbf{1 + ( 1 - a _ { i j } ) ^ { 2 }}}$

Since, $\frac{a_{i j}^{2}}{1+\left(1-a_{i j}\right)^{2}} \leq a_{i j}^{2}$ as $1+\left(1-a_{i j}\right)^{2} \geq 1$.

$$
\leq a_{i j}, \text { for all } i \text { and } j \text {. }
$$

Hence, $A \odot_{\varepsilon} A \leq A$.

The following properties are obvious. The operations the Einstein sum $\oplus_{\varepsilon}$ and the Einstein product $\odot_{\varepsilon}$ are commutative as well as associative.

Property 1.3: If $A, B$ and $C$ are three fuzzy matrices of same size, then
(i) $A \bigoplus_{\varepsilon} B=B \bigoplus_{\varepsilon} A$
(ii) $A \bigoplus_{\varepsilon}\left(B \oplus_{\varepsilon} C\right)=\left(A \oplus_{\varepsilon} B\right) \oplus_{\varepsilon} C$
(iii) $A \bigodot_{\varepsilon} B=B \bigodot_{\varepsilon} A$ 
(iv) $A \bigodot_{\varepsilon}\left(B \bigodot_{\varepsilon} C\right)=\left(A \bigodot_{\varepsilon} B\right) \bigodot_{\varepsilon} C$.

Property 1.4: If $A, B$ and $C$ are three fuzzy matrices of same size and if $A \leq B$ then $A \oplus_{\varepsilon} C \leq B \oplus_{\varepsilon} C, A \odot_{\varepsilon} C \leq B \odot_{\varepsilon} C$.

Proof: Let $d_{i j}, e_{i j}, f_{i j}$ and $g_{i j}$ be the $i j^{\text {th }}$ elements of $A \oplus_{\varepsilon} C, B \oplus_{\varepsilon} C, A \odot_{\varepsilon} C$ and $B \odot_{\varepsilon} C$ respectively. Then $d_{i j}=\frac{a_{i j}+c_{i j}}{\left(1+a_{i j} c_{i j}\right)}, e_{i j}=\frac{b_{i j}+c_{i j}}{\left(1+b_{i j} c_{i j}\right)}$

$f_{i j}=\frac{a_{i j} c_{i j}}{1+\left(1-a_{i j}\right)\left(1-c_{i j}\right)}$ and $g_{i j}=\frac{b_{i j} c_{i j}}{1+\left(1-b_{i j}\right)\left(1-c_{i j}\right)}$

Since $A \leq B, a_{i j} \leq b_{i j}$ Then,$a_{i j}\left(1-c_{i j}^{2}\right) \leq b_{i j}\left(1-c_{i j}^{2}\right)$

$a_{i j}+b_{i j} c_{i j}^{2} \leq b_{i j}+a_{i j} c_{i j}^{2}$

$a_{i j}+b_{i j} c_{i j}^{2}+\left(1+a_{i j} b_{i j}\right) c_{i j} \leq b_{i j}+a_{i j} c_{i j}^{2}+\left(1+a_{i j} b_{i j}\right) c_{i j}$

$a_{i j}+c_{i j}+\left(a_{i j}+c_{i j}\right) b_{i j} c_{i j} \leq b_{i j}+c_{i j}+\left(b_{i j}+c_{i j}\right) a_{i j} c_{i j}$

$\left(a_{i j}+c_{i j}\right)\left(1+b_{i j} c_{i j}\right) \leq\left(b_{i j}+c_{i j}\right)\left(1+a_{i j} c_{i j}\right)$

$\frac{a_{i j}+c_{i j}}{\left(1+a_{i j} c_{i j}\right)} \leq \frac{b_{i j}+c_{i j}}{\left(1+b_{i j} c_{i j}\right)}$

That is, $d_{i j} \leq e_{i j}$, for all $i$ and $j$.

Hence, $A \oplus_{\varepsilon} C \leq B \oplus_{\varepsilon} C$.

Again, $a_{i j} c_{i j} \leq b_{i j} c_{i j}$

$a_{i j} c_{i j}\left(2-c_{i j}\right) \leq b_{i j} c_{i j}\left(2-c_{i j}\right)$

$a_{i j} c_{i j}\left(2-c_{i j}\right)-a_{i j} b_{i j} c_{i j}\left(1-c_{i j}\right) \leq b_{i j} c_{i j}\left(2-c_{i j}\right)-$

$a_{i j} b_{i j} c_{i j}\left(1-c_{i j}\right)$

$a_{i j} c_{i j}\left(2-c_{i j}-b_{i j}\left(1-c_{i j}\right)\right) \leq b_{i j} c_{i j}\left(2-c_{i j}-a_{i j}\left(1-c_{i j}\right)\right)$

$a_{i j} c_{i j}\left(1+\left(1-b_{i j}\right)\left(1-c_{i j}\right)\right) \leq b_{i j} c_{i j}\left(1+\left(1-a_{i j}\right)(1-\right.$

$\left.\left.c_{i j}\right)\right)$

$\frac{a_{i j} c_{i j}}{1+\left(1-a_{i j}\right)\left(1-c_{i j}\right)} \leq \frac{b_{i j} c_{i j}}{1+\left(1-b_{i j}\right)\left(1-c_{i j}\right)}$

That is, $f_{i j} \leq g_{i j}$, for all $i$ and $j$.

Hence, $A \odot_{\varepsilon} C \leq B \odot_{\varepsilon} C$.

The operations the Einstein sum $\bigoplus_{\varepsilon}$ and the Einstein product $\bigodot_{\varepsilon}$ do not obey the De Morgan's laws (over transpose).

(i) $\left(A \oplus_{\varepsilon} B\right)^{T}=A^{T} \oplus_{\varepsilon} B^{T}$

(ii) $\left(A \bigodot_{\varepsilon} B\right)^{T}=A^{T} \bigodot_{\varepsilon} B^{T}$, where $A^{T}$ is the transpose of $A$.

\section{Results on complement of fuzzy matrix}

The complement of a fuzzy matrix is used to analysis the complement nature of any system. For example if $A$ represents the crowdness of a network at a particular time period then its complement $A^{C}$ represents the cleamess at the same time period. Using the following results we can study the complement nature of a system with the help of original fuzzy matrix.

The operator complement obey the De Morgan's laws for the operations the Einstein sum $\oplus_{\varepsilon}$ and the Einstein product $\odot_{\varepsilon}$. This is established in the following property.

Property 2.1: For any fuzzy matrices $A$ and $B$ of same size,

(i) $\left(A \oplus_{\varepsilon} B\right)^{C}=A^{C} \odot_{\varepsilon} B^{C}$

(ii) $\left(A \odot_{\varepsilon} B\right)^{C}=A^{C} \oplus_{\varepsilon} B^{C}$

(iii) $\left(A \oplus_{\varepsilon} B\right)^{C} \leq A^{C} \oplus_{\varepsilon} B^{C}$

(iv) $\left(A \odot_{\varepsilon} B\right)^{C} \geq A^{C} \odot_{\varepsilon} B^{C}$, where $A^{C}$ is the complement of A.

Proof: $(i)$ The $i j^{\text {th }}$ element of $\left(A \oplus_{\varepsilon} B\right)^{C}$ is

$1-\frac{a_{i j} b_{i j}}{1+\left(1-a_{i j}\right)\left(1-b_{i j}\right)}=\frac{2-a_{i j}-b_{i j}}{1+\left(1-a_{i j}\right)\left(1-b_{i j}\right)}$ is equal the

$i j^{\text {th }}$ element of $A^{C} \bigodot_{\varepsilon} B^{C}$. Hence $(i)$ is hold.

(ii) The $i j^{\text {th }}$ element of $\left(A \bigodot_{\varepsilon} B\right)^{C}$ is

$1-\frac{a_{i j}+b_{i j}}{\left(1+a_{i j} b_{i j}\right)}=\frac{\left(1-a_{i j}\right)\left(1-b_{i j}\right)}{\left(1+a_{i j} b_{i j}\right)}$ is equal the $i j^{\text {th }}$ element of $A^{C} \oplus_{\varepsilon} B^{C}$.

Hence ( $i i$ ) is hold.

(iii) From Property 1.1 A $\oplus_{\varepsilon} B \geq A \odot_{\varepsilon} B$.

Then $\left(A \oplus_{\varepsilon} B\right)^{C} \leq\left(A \bigodot_{\varepsilon} B\right)^{C}$

$$
=A^{C} \bigoplus_{\varepsilon} B^{C} \text {. }
$$

(iii) From Property 1.1, $A \oplus_{\varepsilon} B \geq A \odot_{\varepsilon} B$.

Then $\left(A \odot_{\varepsilon} B\right)^{C} \geq\left(A \oplus_{\varepsilon} B\right)^{C}$

$$
=A^{C} \bigodot_{\varepsilon} B^{C} \text {. }
$$

Theorem 2.1: For any fuzzy matrices $A$ and $B$ of same size,

(i) $(A \wedge B) \bigoplus_{\varepsilon}(A \vee B)=A \bigoplus_{\varepsilon} B$

(ii) $(A \wedge B) \bigodot_{\varepsilon}(A \vee B)=A \odot_{\varepsilon} B$

Proof:

(i) $(A \wedge B) \oplus_{\varepsilon}(A \vee B)=\left[\min \left(a_{i j}, b_{i j}\right)\right] \oplus_{\varepsilon}\left[\max \left(a_{i j}, b_{i j}\right)\right]$ $=\left[\frac{\min \left(a_{i j}, b_{i j}\right)+\max \left(a_{i j}, b_{i j}\right)}{1+\min \left(a_{i j}, b_{i j}\right) \max \left(a_{i j}, b_{i j}\right)}\right]$ $=\left[\frac{a_{i j}+b_{i j}}{1+a_{i j} b_{i j}}\right]$ $=A \oplus_{\varepsilon} B$.

(ii) $(A \wedge B) \bigodot_{\varepsilon}(A \vee B)=\left[\min \left(a_{i j}, b_{i j}\right)\right] \odot_{\varepsilon}\left[\max \left(a_{i j}, b_{i j}\right)\right]$ $=\left[\frac{\min \left(a_{i j}, b_{i j}\right) \max \left(a_{i j}, b_{i j}\right)}{1+\left(1-\min \left(a_{i j}, b_{i j}\right)\right)\left(1-\max \left(a_{i j}, b_{i j}\right)\right)}\right]$ $=\left[\frac{a_{i j} b_{i j}}{1+\max \left(1-a_{i j}, 1-b_{i j}\right) \min \left(1-a_{i j}, 1-b_{i j}\right)}\right]$ $=\left[\frac{a_{i j} b_{i j}}{1+\left(1-a_{i j}\right)\left(1-b_{i j}\right)}\right]$ $=A \odot_{\varepsilon} B$.

\section{Intuitionistic fuzzy matrices}

Definition: If $A=\left[a_{i j}, a_{i j}^{\prime}\right]$ and $B=\left[b_{i j}, b_{i j}^{\prime}\right]$ are two intuitionistic fuzzy matrices (IFMs) of same size, then

(i) The Einstein sum of $A$ and $B$ is defined by

$$
A \oplus_{\varepsilon} B=\left[\frac{\mathrm{a}_{\mathrm{ij}}+\mathrm{b}_{\mathrm{ij}}}{1+\mathrm{a}_{\mathrm{ij}} \mathrm{b}_{\mathrm{ij}}}, \frac{\mathrm{a}_{\mathrm{ij}}^{\prime} \mathrm{b}_{\mathrm{ij}}^{\prime}}{1+\left(1-\mathrm{a}_{\mathrm{ij}}^{\prime}\right)\left(1-\mathrm{b}_{\mathrm{ij}}^{\prime}\right)}\right]
$$

(ii) The Einstein product of $A$ and $B$ is defined by

$$
A \odot_{\varepsilon} B=\left[\frac{a_{i j} b_{i j}}{1+\left(1-a_{i j}\right)\left(1-b_{i j}\right)}, \frac{\mathrm{a}_{\mathrm{ij}}^{\prime} \mathrm{b}_{\mathrm{ij}}^{\prime}}{1+\mathrm{a}_{\mathrm{ij}}^{\prime} \mathrm{b}_{\mathrm{ij}}^{\prime}}\right]
$$

Lemma 3.1 Let $a, b \in[0,1]$, then maximum of $\left[\frac{a b}{1+(1-a)(1-b)}, \frac{\mathrm{a}+\mathrm{b}}{1+\mathrm{ab}}\right]=\frac{\mathrm{a}+\mathrm{b}}{1+\mathrm{ab}}$

Property 3.1: If $A$ and $B$ are two fuzzy matrices of same size, then $A \oplus_{\varepsilon} B \geq A \bigodot_{\varepsilon} B$.

Proof: The $i j^{\text {th }}$ element of $A \bigoplus_{\varepsilon} B$ is $\left(\frac{a_{i j}+b_{i j}}{1+a_{i j} b_{i j}}, \frac{a_{i j}^{\prime} b_{i j}^{\prime}}{1+\left(1-a_{i j}^{\prime}\right)\left(1-b_{i j}^{\prime}\right)}\right)$ and that of $A \bigodot_{\varepsilon} B$ is $\left(\frac{a_{i j} b_{i j}}{1+\left(1-a_{i j}\right)\left(1-b_{i j}\right)}, \frac{\mathrm{a}_{\mathrm{ij}}^{\prime} \mathrm{b}_{\mathrm{ij}}^{\prime}}{1+\mathrm{a}_{\mathrm{ij}}^{\prime} \mathrm{b}_{\mathrm{ij}}^{\prime}}\right)$. By lemma 3.1 $\frac{\mathrm{a}_{\mathrm{ij}}+\mathrm{b}_{\mathrm{ij}}}{1+\mathrm{a}_{\mathrm{ij}} \mathrm{b}_{\mathrm{ij}}} \geq \frac{a_{i j} b_{i j}}{1+\left(1-a_{i j}\right)\left(1-b_{i j}\right)}$ and $\frac{a_{i j}^{\prime} b_{i j}^{\prime}}{1+\left(1-a_{i j}^{\prime}\right)\left(1-b_{i j}^{\prime}\right)} \leq \frac{a_{i j}^{\prime} b_{i j}^{\prime}}{1+a_{i j}^{\prime} b_{i j}^{\prime}}$ for all $i$

and $j$

Hence, $A \oplus_{\varepsilon} B \geq A \bigodot_{\varepsilon} B$.

Property 3.2: For any fuzzy matrix $A$,

(i) $A \oplus_{\varepsilon} A \geq A$

(ii) $A \bigodot_{\varepsilon} A \leq A$

Proof: $(i)$ The $i j^{\text {th }}$ element of $A \bigoplus_{\varepsilon} A$ is $\left(\frac{\mathrm{a}_{\mathrm{ij}}+\mathrm{a}_{\mathrm{ij}}}{1+\mathrm{a}_{\mathrm{ij}}{ }^{2}}, \frac{\mathrm{a}_{\mathrm{ij}}{ }^{2}}{1+\left(1-\mathrm{a}_{\mathrm{ij}}^{\prime}\right)^{2}}\right)$

Since $2 \geq\left(1+a_{i j}^{2}\right)$

$$
\begin{gathered}
2 a_{i j} \geq a_{i j}\left(1+a_{i j}^{2}\right) \\
\frac{2 a_{i j}}{1+a_{i j}^{2}} \geq a_{i j}, \text { for all } i \text { and } j .
\end{gathered}
$$

Also $\frac{\mathrm{a}_{\mathrm{ij}}^{\prime 2}}{1+\left(1-\mathrm{a}_{\mathrm{ij}}^{\prime}\right)^{2}} \leq a_{i j}^{\prime 2} \leq a_{i j}^{\prime}$ for all $\mathrm{i}$ and $\mathrm{j}$.

Hence, $A \oplus_{\varepsilon} A \geq A$.

(ii) It can proved similarly.

Note : $\left(A \bigoplus_{\varepsilon} A\right)=\left[\frac{\mathrm{a}_{\mathrm{ij}}+\mathrm{a}_{\mathrm{ij}}}{1+\mathrm{a}_{\mathrm{ij}}^{2}}, \frac{\mathrm{a}_{\mathrm{ij}}{ }^{2}}{1+\left(1-\mathrm{a}_{\mathrm{ij}}\right)^{2}}\right]$

$$
\begin{aligned}
2 A & =\left[\frac{\left(1+a_{i j}\right)^{2}-\left(1-a_{i j}\right)^{2}}{\left(1+a_{i j}\right)^{2}+\left(1-a_{i j}\right)^{2}}, \frac{2 a_{i j}{ }^{\prime 2}}{\left(2-a_{i j}^{\prime}\right)^{2}+a_{i j}{ }^{2}}\right] \\
3 A & =\left[\frac{\left(1+a_{i j}\right)^{3}-\left(1-a_{i j}\right)^{3}}{\left(1+a_{i j}\right)^{3}+\left(1-a_{i j}\right)^{3}}, \frac{2 a_{i j}{ }^{3}}{\left(2-a_{i j}^{\prime}\right)^{3}+a_{i j}{ }^{3}}\right]
\end{aligned}
$$

Similarly 
In general $\quad n A=\left[\frac{\left(1+a_{i j}\right)^{n}-\left(1-a_{i j}\right)^{n}}{\left(1+a_{i j}\right)^{n}+\left(1-a_{i j}\right)^{n}}, \frac{2 a_{i j}{ }^{n}}{\left(2-a_{i j}\right)^{n}+a_{i j}{ }^{n}}\right]$

\section{Theorem 3.1}

Let $\mathrm{n}$ be any positive integer and $\mathrm{A}$ be a intuitionistic fuzzy matrix then $\mathrm{nA}$ is also an intuitionistic fuzzy matrix.

\section{Proof}

Since $0 \leq a_{i j} \leq 1,0 \leq a_{i j}^{\prime} \leq 1$ and $0 \leq a_{i j}+a_{i j}^{\prime} \leq 1$, then $1-a_{i j} \geq a_{i j}^{\prime} \geq 0,1-a_{i j}^{\prime} \geq a_{i j} \geq 0$ and $\left(1-\mathrm{a}_{\mathrm{ij}}\right)^{\mathrm{n}} \geq a_{i j}^{\prime}{ }^{\mathrm{n}}$. We have

$$
\begin{aligned}
\frac{\left(1+a_{i j}\right)^{n}-\left(1-a_{i j}\right)^{n}}{\left(1+a_{i j}\right)^{n}+\left(1-a_{i j}\right)^{n}} & \leq \frac{\left(1+a_{i j}\right)^{n}-a_{i j}^{\prime}{ }^{n}}{\left(1+a_{i j}\right)^{n}+a_{i j}^{\prime}{ }^{n}} \text { and } \\
\frac{2 a_{i j}{ }^{n}}{\left(2-a_{i j}^{\prime}\right)^{n}+a_{i j}{ }^{n}} & \leq \frac{2 a_{i j}{ }^{n}}{\left(1+a_{i j}^{\prime}\right)^{n}+a_{i j}{ }^{n}}
\end{aligned}
$$

$\frac{\left(1+a_{i j}\right)^{n}-\left(1-a_{i j}\right)^{n}}{\left(1+a_{i j}\right)^{n}+\left(1-a_{i j}\right)^{n}}+\frac{2 a_{i j}{ }^{n}}{\left(2-a_{i j}^{\prime}\right)^{n}+a_{i j}{ }^{n}} \leq 1$

Furthermore, we have

$$
\begin{aligned}
& \frac{\left(1+\mathrm{a}_{\mathrm{ij}}\right)^{\mathrm{n}}-\left(1-\mathrm{a}_{\mathrm{ij}}\right)^{\mathrm{n}}}{\left(1+\mathrm{a}_{\mathrm{ij}}\right)^{\mathrm{n}}+\left(1-\mathrm{a}_{\mathrm{ij}}\right)^{\mathrm{n}}}+\frac{2 \mathrm{a}_{\mathrm{ij}}{ }^{\mathrm{n}}}{\left(2-\mathrm{a}_{\mathrm{ij}}\right)^{\mathrm{n}}+\mathrm{a}_{\mathrm{ij}}{ }^{\mathrm{n}}}=1 \text { iff } a_{i j}=a_{i j}^{\prime}=0 . \\
& \frac{\left(1+\mathrm{a}_{\mathrm{ij}}\right)^{\mathrm{n}}-\left(1-\mathrm{a}_{\mathrm{ij}}\right)^{\mathrm{n}}}{\left(1+\mathrm{a}_{\mathrm{ij}}\right)^{\mathrm{n}}+\left(1-\mathrm{a}_{\mathrm{ij}}\right)^{\mathrm{n}}}+\frac{2 \mathrm{a}_{\mathrm{ij}}{ }^{\prime}}{\left(2-\mathrm{a}_{\mathrm{ij}}^{\prime}\right)^{\mathrm{n}}+\mathrm{a}_{\mathrm{ij}}{ }^{\mathrm{n}}}=0 \text { iff } a_{i j}+a_{i j}^{\prime}=1
\end{aligned}
$$

\section{Theorem 3.2}

Let $\mathrm{A}$ and $\mathrm{B}$ be intuitionistic fuzzy matrices of same order and $n_{1}$ $, n_{2}, \mathrm{n}>0$ be positive integers, then
(i) $n\left(A \oplus_{\varepsilon} B\right)=n A \oplus_{\varepsilon} n B$.
(ii) $\left(n_{1} A \oplus_{\varepsilon} n_{2} A\right)=\left(n_{1}+n_{2}\right) A$.
(iii) $\left(n_{1} n_{2}\right) \mathrm{A}=n_{1}\left(n_{2} \mathrm{~A}\right)$.

\section{Proof}

$$
\begin{aligned}
(A & \left.\oplus_{\varepsilon} B\right)=\left[\frac{a_{i j}+b_{i j}}{1+a_{i j} b_{i j}}, \frac{a_{i j}^{\prime} b_{i j}^{\prime}}{1+\left(1-a_{i j}^{\prime}\right)\left(1-b_{i j}^{\prime}\right)}\right] \\
= & {\left[\frac{\left(1+a_{i j}\right)\left(1+b_{i j}\right)-\left(1-a_{i j}\right)\left(1-b_{i j}\right)}{\left(1+a_{i j}\right)\left(1+b_{i j}\right)+\left(1-a_{i j}\right)\left(1-b_{i j}\right)}, \frac{2 a_{i j}^{\prime} b_{i j}^{\prime}}{\left(2-a_{i j}^{\prime}\right)\left(2-b_{i j}^{\prime}\right)+a_{i j}^{\prime} b_{i j}^{\prime}}\right] }
\end{aligned}
$$

$$
\left(1+a_{i j}\right)\left(1+b_{i j}\right)=c_{i j} \quad\left(1-a_{i j}\right)\left(1-b_{i j}\right)=d_{i j}
$$$$
\left(2-\mathrm{a}_{\mathrm{ij}}^{\prime}\right)\left(2-\mathrm{b}_{\mathrm{ij}}^{\prime}\right)=e_{i j} \text { and } \mathrm{a}_{\mathrm{ij}}^{\prime} \mathrm{b}_{\mathrm{ij}}^{\prime}=f_{i j}
$$

$$
\left(A \oplus_{\varepsilon} B\right)=\left[\frac{\mathrm{c}_{\mathrm{ij}}-\mathrm{d}_{\mathrm{ij}}}{\mathrm{c}_{\mathrm{ij}}+\mathrm{d}_{\mathrm{ij}}}, \frac{2 \mathrm{e}_{\mathrm{ij}}}{\mathrm{f}_{\mathrm{ij}}+\mathrm{e}_{\mathrm{ij}}}\right]
$$

By (1), it follows that

$$
\begin{aligned}
& n\left(A \oplus_{\varepsilon} B\right)= \\
& {\left[\frac{\left(1+\frac{c_{i j}-d_{i j}}{c_{i j}+d_{i j}}\right)^{n}-\left(1-\frac{c_{i j}-d_{i j}}{c_{i j}+d_{i j}}\right)^{n}}{\left(1+\frac{c_{i j}-d_{i j}}{c_{i j}+d_{i j}}\right)^{n}+\left(1-\frac{c_{i j}-d_{i j}}{c_{i j}+d_{i j}}\right)^{n}}, \frac{2\left(\frac{2 e_{i j}}{f_{i j}+e_{i j}}\right)^{n}}{\left(2+\frac{2 e_{i j}}{f_{i j}+e_{i j}}\right)^{n}+\left(\frac{2 e_{i j}}{f_{i j}+e_{i j}}\right)^{n}}\right]} \\
& =\left[\frac{c_{i j}^{n}-d_{i j}^{n}}{c_{i j}^{n}+d_{i j}^{n}}, \frac{2 e_{i j}^{n}}{f_{i j}^{n}+e_{i j}^{n}}\right] \\
& = \\
& {\left[\frac{\left(1+a_{i j}\right)^{n}\left(1+b_{i j}\right)^{n}-\left(1-a_{i j}\right)^{n}\left(1-b_{i j}\right)^{n}}{\left(1+a_{i j}\right)^{n}\left(1+b_{i j}\right)^{n}+\left(1-a_{i j}\right)^{n}\left(1-b_{i j}\right)^{n}}, \frac{2 a_{i j}{ }^{n} b_{i j}{ }^{n}}{\left(2-a_{i j}^{\prime}\right)^{n}\left(2-b_{i j}^{\prime}\right)^{n}+a_{i j}{ }^{n} b_{i j}{ }^{n}}\right]} \\
& \text { Since, } n A=\left[\frac{\left(1+a_{i j}\right)^{n}-\left(1-a_{i j}\right)^{n}}{\left(1+a_{i j}\right)^{n}+\left(1-a_{i j}\right)^{n}}, \frac{2 a_{i j}{ }^{n}}{\left(2-a_{i j}^{\prime}\right)^{n}+a_{i j}{ }^{n}}\right] \\
& \text { and } n B=\left[\frac{\left(1+b_{i j}\right)^{n}-\left(1-b_{i j}\right)^{n}}{\left(1+b_{i j}\right)^{n}+\left(1-b_{i j}\right)^{n}}, \frac{2 b_{i j}{ }^{, n}}{\left(2-b_{i j}{ }^{n}{ }^{n}+b_{i j}{ }^{n}\right.}\right] \\
& \mathrm{g}_{\mathrm{ij}}=\left(1+\mathrm{a}_{\mathrm{ij}}\right)^{\mathrm{n}}, \mathrm{h}_{\mathrm{ij}}=\left(1-\mathrm{a}_{\mathrm{ij}}\right)^{\mathrm{n}}, m_{i j}=\left(2-\mathrm{a}_{\mathrm{ij}}^{\prime}\right)^{\mathrm{n}}, \mathrm{k}_{\mathrm{ij}}= \\
& a_{i j}{ }^{\prime n} \\
& n_{i j}=\left(1+b_{i j}\right)^{n}, \quad p_{i j}=\left(1-b_{i j}\right)^{n}, \quad r_{i j}=\left(2-b_{i j}^{\prime}\right)^{n}, q_{i j}= \\
& \mathrm{b}_{\mathrm{ij}}{ }^{\mathrm{n}} \text {. }
\end{aligned}
$$

By the definition of Einstein sum,

$$
\begin{gathered}
\left(n A \oplus_{\varepsilon} n B\right)=\left[\frac{g_{i j}^{n}-h_{i j}^{n}}{g_{i j}^{n}+h_{i j}^{n}}, \frac{2 k_{i j}^{n}}{m_{i j}^{n}+k_{i j}^{n}}\right] \\
\bigoplus_{\varepsilon}\left[\frac{n_{i j}^{n}-p_{i j}^{n}}{n_{i j}^{n}+p_{i j}^{n}}, \frac{2 q_{i j}^{n}}{r_{i j}^{n}+q_{i j}^{n}}\right] \\
=\left[\frac{\left(\frac{g_{i j}-h_{i j}}{g_{i j}+h_{i j}}\right)-\left(\frac{n_{i j}-p_{i j}}{n_{i j}+p_{i j}}\right)}{1+\left(\frac{g_{i j}-d_{i j}}{g_{i j}+d_{i j}}\right)\left(\frac{n_{i j}-p_{i j}}{n_{i j}+p_{i j}}\right)}, \frac{\left(\frac{2 k_{i j}}{m_{i j}+k_{i j}}\right)\left(\frac{2 q_{i j}}{r_{i j}+a_{i j}}\right)}{1+\left(1-\frac{2 k_{i j}}{c_{i j}+d_{i j}}\right)\left(1-\frac{2 q_{i j}}{c_{i j}+d_{i j}}\right)}\right] \\
=\left[\frac{g_{i j} n_{i j}-h_{i j} p_{i j}}{g_{i j} n_{i j}+h_{i j} p_{i j}}, \frac{2 k_{i j} q_{i j}}{m_{i j} r_{i j}+k_{i j} a_{i j}}\right] \\
=\left[\frac{\left(1+a_{i j}\right)^{n}\left(1+b_{i j}\right)^{n}-\left(1-a_{i j}\right)^{n}\left(1-b_{i j}\right)^{n}}{\left(1+a_{i j}\right)^{n}\left(1+b_{i j}\right)^{n}+\left(1-a_{i j}\right)^{n}\left(1-b_{i j}\right)^{n}}, \frac{2 a_{i j} b^{n}}{\left(2-a_{i j}\right)^{n}\left(2-b_{i j}{ }^{\prime}\right)^{n}+a_{i j}{ }^{n} b_{i j}{ }^{n}{ }^{n}}\right]
\end{gathered}
$$

Hence $n\left(A \oplus_{\varepsilon} B\right)=n A \oplus_{\varepsilon} n B$.

(ii) Since $n_{1} \mathrm{~A}=\left[\frac{\left(1+a_{i j}\right)^{n_{1}}-\left(1-a_{i j}\right)^{n_{1}}}{\left(1+a_{i j}\right)^{n_{1}}+\left(1-a_{i j}\right)^{n_{1}}}, \frac{2 a_{i j}{ }^{n_{1}}}{\left(2-a_{i j}^{\prime}\right)^{n_{1}}+a_{i j}{ }^{n_{1}}}\right]$

$$
n_{2} \mathrm{~A}=\left[\frac{\left(1+a_{i j}\right)^{n_{2}}-\left(1-a_{i j}\right)^{n_{2}}}{\left(1+a_{i j}\right)^{n_{2}+\left(1-a_{i j}\right)^{n_{2}}}}, \frac{2 a_{i j}{ }^{n_{2}}}{\left(2-a_{i j}^{\prime}\right)^{n_{2}}+a_{i j}{ }^{n_{2}}}\right]
$$

$n_{1}, n_{2}>0, c_{\mathrm{ij}}=\left(1+\mathrm{a}_{\mathrm{ij}}\right)^{\mathrm{n}_{1}}, \mathrm{~d}_{\mathrm{ij}}=\left(1-\mathrm{a}_{\mathrm{ij}}\right)^{\mathrm{n}_{1}}, f_{i j}=$ $\left(2-a_{i j}^{\prime}\right)^{n_{1}}, e_{i j}=a_{i j}{ }^{n_{1}}$ $\mathrm{a}_{\mathrm{ij}}{ }^{\mathrm{n}}$

$$
\mathrm{g}_{\mathrm{ij}}=\left(1+\mathrm{a}_{\mathrm{ij}}\right)^{\mathrm{n}_{2}}, \quad \mathrm{~h}_{\mathrm{ij}}=\left(1-\mathrm{a}_{\mathrm{ij}}\right)^{\mathrm{n}_{2}}, l_{i j}=\left(2-\mathrm{a}_{\mathrm{ij}}^{\prime}\right)^{\mathrm{n}_{2}}, \mathrm{k}_{\mathrm{ij}}=
$$

$$
\begin{aligned}
& n_{1} \mathrm{~A}=\left[\frac{c_{i j}-d_{i j}}{c_{i j}+d_{i j}}, \frac{2 e_{i j}}{f_{i j}+e_{i j}}\right] \\
& n_{2} \mathrm{~A}=\left[\frac{g_{i j}-h_{i j}}{g_{i j}+h_{i j}}, \frac{2 k_{i j}}{l_{i j}+e_{i j}}\right]
\end{aligned}
$$

By the definition of Einstein sum, it follows that

$$
\begin{gathered}
\left(n_{1} A \oplus_{\varepsilon} n_{2} A\right)=\left[\frac{\left(\frac{c_{i j}-d_{i j}}{c_{i j}+d_{i j}}\right)-\left(\frac{g_{i j}-h_{i j}}{g_{i j}+h_{i j}}\right)}{1+\left(\frac{c_{i j}-d_{i j}}{c_{i j}+d_{i j}}\right)\left(\frac{g_{i j}-h_{i j}}{g_{i j}+h_{i j}}\right)}, \frac{\left(\frac{2 e_{i j}}{f_{i j}+e_{i j}}\right)\left(\frac{2 k_{i j}}{l_{i j}+k_{i j}}\right)}{1+\left(1-\frac{2 e_{i j}}{f_{i j}+e_{i j}}\right)\left(1-\frac{2 k_{i j}}{l_{i j}+k_{i j}}\right)}\right] \\
=\left[\frac{c_{i j} g_{i j}-d_{i j} h_{i j}}{c_{i j} g_{i j}+d_{i j} h_{i j}}, \frac{2 e_{i j} k_{i j}}{f_{i j} l_{i j}+e_{i j} k_{i j}}\right] \\
=\left[\frac{\left(1+a_{i j}\right)^{n_{1}+n_{2}}-\left(1-a_{i j}\right)^{n_{1}+n_{2}}}{\left(1+a_{i j}\right)^{n_{1}+n_{2}}+\left(1-a_{i j}\right)^{n_{1}+n_{2}}}, \frac{2 a_{i j}{ }^{{ }^{n_{1}+n_{2}}}}{\left(2-a_{i j}^{\prime}\right)^{n_{1}+n_{2}}+a_{i j}{ }^{{ }^{n} 1+n_{2}}}\right]
\end{gathered}
$$

$\left(n_{1} A \oplus_{\varepsilon} n_{2} A\right)=\left(n_{1}+n_{2}\right) A$.

(iii) Since $n_{2} \mathrm{~A}=\left[\frac{\left(1+a_{i j}\right)^{n_{2}}-\left(1-a_{i j}\right)^{n_{2}}}{\left(1+a_{i j}\right)^{n_{2}}+\left(1-a_{i j}\right)^{n_{2}}}, \frac{2 a_{i j}{ }^{n_{2}}}{\left(2-a_{i j}^{\prime}\right)^{n_{2}}+a_{i j}{ }^{n_{2}}}\right]$

Let $c_{i j}=\left(1+a_{i j}\right)^{n_{2}}, \quad d_{i j}=\left(1-a_{i j}\right)^{n_{2}}, f_{i j}=(2-$

$\left.a_{i j}^{\prime}\right)^{n_{2}}, e_{i j}=a_{i j}{ }^{{ }^{n}}{ }^{2}$

then $\quad n_{2} \mathrm{~A}=\left[\frac{c_{i j}-d_{i j}}{c_{i j}+d_{i j}}, \frac{2 e_{i j}}{f_{i j}+e_{i j}}\right]$

$$
\begin{aligned}
& n_{1}\left(n_{2} A\right)= \\
& {\left[\frac{\left(1+\frac{c_{i j}-d_{i j}}{c_{i j}+d_{i j}}\right)^{n_{1}}-\left(1-\frac{c_{i j}-d_{i j}}{c_{i j}+d_{i j}}\right)^{n_{1}}}{\left(1+\frac{c_{i j}-d_{i j}}{c_{i j}+d_{i j}}\right)^{n_{1}}+\left(1-\frac{c_{i j}-d_{i j}}{c_{i j}+d_{i j}}\right)^{n_{1}}}, \frac{2\left(\frac{2 e_{i j}}{f_{i j}+e_{i j}}\right)^{n_{1}}}{\left(2+\frac{2 e_{i j}}{f_{i j}+e_{i j}}\right)^{n_{1}}+\left(\frac{2 e_{i j}}{f_{i j}+e_{i j}}\right)^{n_{1}}}\right]} \\
& =\left[\frac{c_{i j}^{n_{1}}-d_{i j}^{n_{1}}}{c_{i j}^{n_{1}}+d_{i j}^{n_{1}}}, \frac{2 e_{i j}^{n_{1}}}{f_{i j}^{n_{1}}+e_{i j}^{n_{1}}}\right] \\
& =\left[\frac{\left(1+a_{i j}\right)^{n_{1} n_{2}}-\left(1-a_{i j}\right)^{n_{1} n_{2}}}{\left(1+a_{i j}\right)^{n_{1} n_{2}}+\left(1-a_{i j}\right)^{n_{1} n_{2}}}, \frac{2 a_{i j}{ }^{n_{1} n_{2}}}{\left(2-a_{i j}^{\prime}\right)^{n_{1} n_{2}}+a_{i j}{ }^{n_{1} n_{2}}}\right] \\
& =\left(n_{1} n_{2}\right) A
\end{aligned}
$$

\section{References}

[1] Atanassov K, "Instuitionistic fuzzy sets", Fuzzy sets and systems, Vol.20, No.1, (1986), pp.87-96.

[2] Kim KH \& Roush FW, "Generalized fuzzy matrices", Fuzzy sets and systems, Vol.4, (1980), pp.293-315.

[3] Oh KW \& Bandler W, "Properties of fuzzy implication operators", Int.J.of Approxi-mate Reasoning, Vol.1, No.13, (1987), pp.273-285.

[4] Ragab MZ \& Emam EG, "On the min-max composition of fuzzy matrices”, Fuzzy Sets and Systems, Vol.75, (1995), pp.83-92. 
[5] Shyamal AK \& Pal M, "Two new operators on fuzzy matrices", $J$. Appl. Math. Computing, Vol.15, No.1-2, (2004), pp.91-107.

[6] Thomason MG, "Convergence of powers of a fuzzy matrix", $J$. Math. Anal. Appl., Vol.57, (1977), pp. 476-480.

[7] Wang W \& Liu X, "Intuitionistic Fuzzy Information Aggregation using Einstein operations", IEEE Transactions on Fuzzy systems, Vol.20, No.5, (2012), pp.923-938.

[8] Zadeh LA, "Fuzzy sets", Information and Control, Vol.8, (1965), pp.338-353 